\title{
A NEW FIVE-POINT BINARY SUBDIVISION SCHEME WITH A PARAMETER
}

YAN WANG* and ZHIMING LI

School of Mathematics and Statistics

Hefei Normal University

Hefei 230601

P. R. China

e-mail:wy030515@163.com

School of Computer and Information

Hefei University of Technology

Hefei 230009

P. R. China

\begin{abstract}
A new five-point binary subdivision scheme with a parameter is proposed. By choice of appropriate parameters, some existing subdivision schemes such as the four-point binary interpolating scheme, the quartic B-spline scheme, and a class of five-point binary approximating scheme can be obtained. It is shown that the resulting curves are $C^{2}$ continuous for the certain range of the parameter.
\end{abstract}

2010 Mathematics Subject Classification: 65D17, 40A05.

Keywords and phrases: binary subdivision, smoothness.

${ }^{*}$ Corresponding Author.

Received May 30, 2018

(C) 2018 Scientific Advances Publishers 


\section{Introduction}

Subdivision method has become a powerful tool to create curves and surfaces in many fields such as in CAGD, CG, and geometric modelling because of its efficiency and simplicity. In accordance with whether the limit curve and surface pass through the initial control vertex, subdivision can be divided into interpolatory subdivision and approximating subdivision.

The typical interpolatory subdivision schemes include the four-point binary interpolating scheme $[1,2]$, the six-point binary interpolating scheme [3], the three-point ternary interpolating scheme [4], the fourpoint ternary interpolating scheme [5], etc. The representative approximating subdivision schemes include Chaikin corner cutting scheme [6] and the B-spline scheme, etc.

Generally speaking, when the length of the mask is equal, the smoothness of the interpolatory subdivision is rarely as good as the approximation subdivision because of the limitation of the interpolation condition. However, interpolatory subdivision can be used to construct the limiting curve passing through all the vertices of the initial polygon. As such, it is very important to study the method of unifying interpolatory subdivision and approximation subdivision. We present a new five-point binary subdivision scheme with parameter in this paper and discuss the continuity of the limit curve. The rest of the paper is organized as follows. Section 2 introduces the preliminaries. In Section 3, a five-point binary subdivision scheme with parameter is introduced. In

Sections 4 , the continuity of the limit curve is discussed. Section 5 concludes the paper with numerical examples. 


\section{Preliminaries}

Given a set of initial control points $P^{0}=\left\{p_{i}^{0}\right\}_{i \in Z}$, let $P^{k}=\left\{p_{i}^{k}\right\}_{i \in Z}$ be the set of control points at level $k(k \geq 0, k \in Z)$. Define $\left\{p_{i}^{k+1}\right\}_{i \in Z}$ recursively by the following binary subdivision rules:

$$
p_{i}^{k+1}=\sum_{j \in z} a_{i-2 j} p_{j}^{k}, i \in Z
$$

where the finite set $a=\left\{a_{i}\right\}_{i \in Z}$ is called the mask. The symbol of the scheme is defined $a(z)=\sum_{i \in z} a_{i} z^{i}$.

Theorem 1 ([7]). Let a binary subdivision scheme $S$ be convergent. Then the mask $a(z)=\sum_{i \in z} a_{i} z^{i}$ satisfies

$$
\sum_{i \in z} a_{2 i}=\sum_{i \in z} a_{2 i+1}=1
$$

Theorem 2 ([7]). Let a subdivision scheme $S$ with a mask $a=\left\{a_{i}\right\}_{i \in Z}$ satisfy (2). Then there exists a subdivision scheme $S_{1}$ (first order difference scheme of $S$ ) which satisfies the property

$$
d P^{k}=S_{1} d P^{k-1}
$$

where $P^{k}=S_{k} P^{0}, d P^{k}=\left\{\left(d P^{k}\right)_{i}=2^{k}\left(p_{i+1}^{k}-p_{i}^{k}\right) \mid i \in Z\right\}$. The symbol of $S_{1}$ is $a^{(1)}(z)=\frac{2 z}{1+z} a(z)$. Generally, if $S_{n}$ exists and $S_{n}$ is the $n$-th order difference scheme of $S$ with a mask $a^{(n)}=\left\{a_{i}^{(n)}\right\}_{i \in Z}$, then the symbol of $S_{n}$ is $a^{(n)}(z)=\sum_{i \in z} a_{i}^{(n)} z^{i}=\left(\frac{2 z}{1+z}\right)^{n} a(z)$. 
Theorem 3 ([8]). Let a subdivision scheme $S$ have a mask $a^{(0)}=\left\{a_{i}^{(0)}\right\}_{i \in Z}$, and its $j$-th order difference scheme $S_{j}(j=1,2, \cdots)$ exist and have the mask $a^{(j)}=\left\{a_{i}^{(j)}\right\}_{i \in Z}$ satisfying

$$
\sum_{i \in Z} a_{2 i}^{(j)}=\sum_{i \in Z} a_{2 i+1}^{(j)}=1, \quad j=0,1,2, \cdots
$$

If there exists an integer $L \geq 1$, such that $\left\|\left(\frac{1}{2} S_{n+1}\right)^{L}\right\|_{\infty}<1$, then the subdivision scheme $S$ is $C^{n}$ continuous, where

$$
\begin{gathered}
\left\|\left(\frac{1}{2} S_{n+1}\right)^{L}\right\|_{\infty}=\left\{\max \sum_{i \in Z}\left|\begin{array}{c}
b_{i-2}^{[L]}(n+1) \\
i{ }^{L}
\end{array}\right|: 0 \leq i<2^{L}\right\}, \\
b^{[L]}(z)=b(z) b\left(z^{2}\right) \cdots, b(z)=\frac{1}{2} a^{(n+1)}(z) .
\end{gathered}
$$

Especially, when $L=1,\left\|\frac{1}{2} S_{n+1}\right\|_{\infty}=\frac{1}{2} \max \left\{\sum_{i \in Z}\left|a_{2 i}^{(n+1)}\right|, \sum_{i \in Z}\left|a_{2 i+1}^{(n+1)}\right|\right\}$.

\section{A New Five-Point Binary Subdivision Scheme}

A new 5-point binary subdivision scheme is defined as follows:

$$
\left\{\begin{array}{l}
P_{2 i}^{k+1}=a_{0} P_{i-2}^{k}+a_{1} P_{i-1}^{k}+a_{2} P_{i}^{k}+a_{3} P_{i+1}^{k}+a_{4} P_{i+2}^{k} \\
P_{2 i+1}^{k+1}=b_{1} P_{i-1}^{k}+b_{2} P_{i}^{k}+b_{3} P_{i+1}^{k}+b_{4} P_{i+2}^{k}
\end{array}\right.
$$

where

$$
\begin{aligned}
a_{0}= & \frac{1}{16} t^{2}-\frac{1}{16} t, a_{1}=-\frac{1}{2} t^{2}+\frac{1}{2} t, a_{2}=-\frac{1}{2} t^{2}-\frac{3}{16} t+1, a_{3}=\frac{3}{4} t^{2}-\frac{1}{8} t, \\
a_{4}= & \frac{3}{16} t^{2}-\frac{1}{8} t \\
b_{1}= & \frac{1}{16} t-\frac{1}{16}, b_{2}=-\frac{7}{8} t^{2}+\frac{3}{8} t+\frac{9}{16}, b_{3}=\frac{1}{4} t^{2}-\frac{3}{16} t+\frac{9}{16}, b_{4}=\frac{5}{8} t^{2}-\frac{1}{4} t \\
& -\frac{1}{16} .
\end{aligned}
$$


And $t$ is a tension parameter. In the case when $t=0$, we can get the scheme by Dubuc [1],

$$
\left\{\begin{array}{l}
P_{2 i}^{k+1}=P_{i}^{k} \\
P_{2 i+1}^{k+1}=-\frac{1}{16} P_{i-1}^{k}+\frac{9}{16} P_{i}^{k}+\frac{9}{16} P_{i+1}^{k}-\frac{1}{16} P_{i+2}^{k} .
\end{array}\right.
$$

When $t=\frac{1}{2}$, the scheme (4) reduces to the quartic B-spline scheme,

$$
\left\{\begin{array}{l}
P_{2 i}^{k+1}=-\frac{1}{64} P_{i-2}^{k}+\frac{1}{8} P_{i-1}^{k}+\frac{25}{32} P_{i}^{k}+\frac{1}{8} P_{i+1}^{k}-\frac{1}{64} P_{i+2}^{k} \\
P_{2 i+1}^{k+1}=-\frac{1}{32} P_{i-1}^{k}+\frac{17}{32} P_{i}^{k}+\frac{17}{32} P_{i+1}^{k}-\frac{1}{32} P_{i+2}^{k} .
\end{array}\right.
$$

When $t=1$, the five-point binary approximating scheme in [9] can be obtained

$$
\left\{\begin{array}{l}
P_{2 i}^{k+1}=\frac{5}{16} P_{i}^{k}+\frac{10}{16} P_{i+1}^{k}+\frac{1}{16} P_{i+2}^{k} \\
P_{2 i+1}^{k+1}=\frac{1}{16} P_{i}^{k}+\frac{10}{16} P_{i+1}^{k}+\frac{5}{16} P_{i+2}^{k}
\end{array}\right.
$$

\section{Smoothness Analysis}

In this section, we use Theorem 3 to analyze the convergence and continuity of the subdivision scheme (4).

Theorem 4. The limiting curve generated by the subdivision scheme (4) is $C^{0}$ continuous in the range $\frac{3-2 \sqrt{11}}{7}<t<\frac{1+\sqrt{89}}{8}, C^{1}$ continuous in the range $0 \leq t<\frac{3+\sqrt{89}}{10}, C^{2}$ continuous in the range $0<t<\frac{-1+\sqrt{33}}{4}$, and $C^{3}$ continuous when $t=\frac{1}{2}, 1$. 
Proof. The generating polynomial for the mask of the subdivision scheme (4) can be written as:

$$
a(z)=a_{0} z^{-4}+b_{1} z^{-3}+a_{1} z^{-2}+b_{2} z^{-1}+a_{2}+b_{3} z+a_{3} z^{2}+b_{4} z^{3}+a_{4} z^{4} .
$$

It is easy to verify that $a(z)$ satisfies Equation (2). According to Theorem 2, we have the generating polynomials for $S_{j}(j=1,2,3,4)$ as follows:

$$
\begin{aligned}
a^{(1)}(z)= & \left(\frac{1}{8} t^{2}-\frac{1}{8} t\right) z^{-3}+\left(-\frac{1}{8} t^{2}+\frac{1}{4} t-\frac{1}{8}\right) z^{-2}+\left(-\frac{7}{8} t^{2}+\frac{3}{4} t+\frac{1}{8}\right) z^{-1} \\
& +\left(-\frac{7}{8} t^{2}+1\right)+\left(-\frac{1}{8} t^{2}-\frac{3}{8} t+1\right) z+\left(\frac{5}{8} t^{2}+\frac{1}{8}\right) z^{2} \\
& +\left(\frac{7}{8} t^{2}-\frac{1}{4} t-\frac{1}{8}\right) z^{3}+\left(\frac{3}{8} t^{2}-\frac{1}{4} t\right) z^{4} \\
a^{(2)}(z)= & \left(\frac{1}{4} t^{2}-\frac{1}{4} t\right) z^{-2}+\left(-\frac{1}{2} t^{2}+\frac{3}{4} t-\frac{1}{4}\right) z^{-1}+\left(-\frac{5}{4} t^{2}+\frac{3}{4} t+\frac{1}{2}\right) \\
& +\left(-\frac{1}{2} t^{2}-\frac{3}{4} t+\frac{3}{2}\right) z+\left(\frac{1}{4} t^{2}+\frac{1}{2}\right) z^{2}+\left(t^{2}-\frac{1}{4}\right) z^{3}+\left(\frac{3}{4} t^{2}-\frac{1}{2} t\right) z^{4}, \\
a^{(3)}(z)= & \left(\frac{1}{2} t^{2}-\frac{1}{2} t\right) z^{-1}+\left(-\frac{3}{2} t^{2}+2 t-\frac{1}{2}\right)+\left(-t^{2}-\frac{1}{2} t+\frac{3}{2}\right) z \\
& +\left(-t+\frac{3}{2}\right) z^{2}+\left(\frac{1}{2} t^{2}+t-\frac{1}{2}\right) z^{3}+\left(\frac{3}{2} t^{2}-t\right) z^{4} \\
a^{(4)}(z)= & \left(t^{2}-t\right)+\left(-4 t^{2}+5 t-1\right) z+\left(2 t^{2}-6 t+4\right) z^{2} \\
& +\left(-2 t^{2}+4 t-1\right) z^{3}+\left(3 t^{2}-2 t\right) z^{4} .
\end{aligned}
$$

It is easy to verify that $a^{(j)}(z)(j=1,2,3)$ satisfy Equation (2).

And when $\frac{3-2 \sqrt{11}}{7}<t<\frac{1+\sqrt{89}}{8}$, we have

$$
\begin{aligned}
\left\|\frac{1}{2} S_{1}\right\|_{\infty}= & \frac{1}{2} \max \left\{\left|\frac{1}{8} t^{2}-\frac{1}{8} t\right|+\left|-\frac{7}{8} t^{2}-\frac{3}{4} t+\frac{1}{8}\right|+\left|-\frac{1}{8} t^{2}-\frac{3}{8} t+1\right|+\left|\frac{7}{8} t^{2}-\frac{1}{4} t-\frac{1}{8}\right|,\right. \\
& \left.\left|-\frac{1}{8} t^{2}+\frac{1}{4} t-\frac{1}{8}\right|+\left|-\frac{7}{8} t^{2}+1\right|+\left|\frac{5}{8} t^{2}+\frac{1}{8}\right|+\left|\frac{3}{8} t^{2}+\frac{1}{4} t\right|\right\}<1 .
\end{aligned}
$$


When $0 \leq t<\frac{3+\sqrt{89}}{10}$, we have

$$
\begin{aligned}
\left\|\frac{1}{2} S_{2}\right\|_{\infty}= & \frac{1}{2} \max \left\{\left|\frac{1}{4} t^{2}-\frac{1}{4} t\right|+\left|-\frac{5}{4} t^{2}+\frac{3}{4} t+\frac{1}{2}\right|+\left|\frac{1}{4} t^{2}+\frac{1}{2}\right|+\left|\frac{3}{4} t^{2}-\frac{1}{2} t\right|,\right. \\
& \left.\left.\left|-\frac{1}{2} t^{2}+\frac{3}{4} t-\frac{1}{4}\right|+\left|-\frac{1}{2} t^{2}-\frac{3}{4} t+\frac{3}{2}\right|+\mid t^{2}-\frac{1}{4}\right\}\right\}<1 .
\end{aligned}
$$

When $0<t<\frac{-1+\sqrt{33}}{4}$, we have

$$
\begin{aligned}
\left\|\frac{1}{2} S_{3}\right\|_{\infty}= & \frac{1}{2} \max \left\{\left|\frac{1}{2} t^{2}-\frac{1}{2} t\right|+\left|-t^{2}-\frac{1}{2} t+\frac{3}{2}\right|+\left|\frac{1}{2} t^{2}+t-\frac{1}{2}\right|,\right. \\
& \left.\left|-\frac{3}{2} t^{2}+2 t-\frac{1}{2}\right|+\left|-t+\frac{3}{2}\right|+\left|\frac{3}{2} t^{2}-t\right|\right\}<1 .
\end{aligned}
$$

When $t=\frac{1}{2}, 1$, we have

$$
\sum_{i \in Z} a_{2 i}^{(4)} \sum_{i \in Z} a_{2 i+1}^{(4)}=1
$$

And when $t=\frac{1}{2},\left\|\left(\frac{1}{2} S_{4}\right)^{2}\right\|_{\infty}=\frac{3}{4}<1$; when $t=1,\left\|\frac{1}{2} S_{4}\right\|_{\infty}=\frac{1}{2} \max \{1,1\}$

$=\frac{1}{2}<1$ 
Consequently, it follows from Theorem 3 that the proof of Theorem 4 is completed.

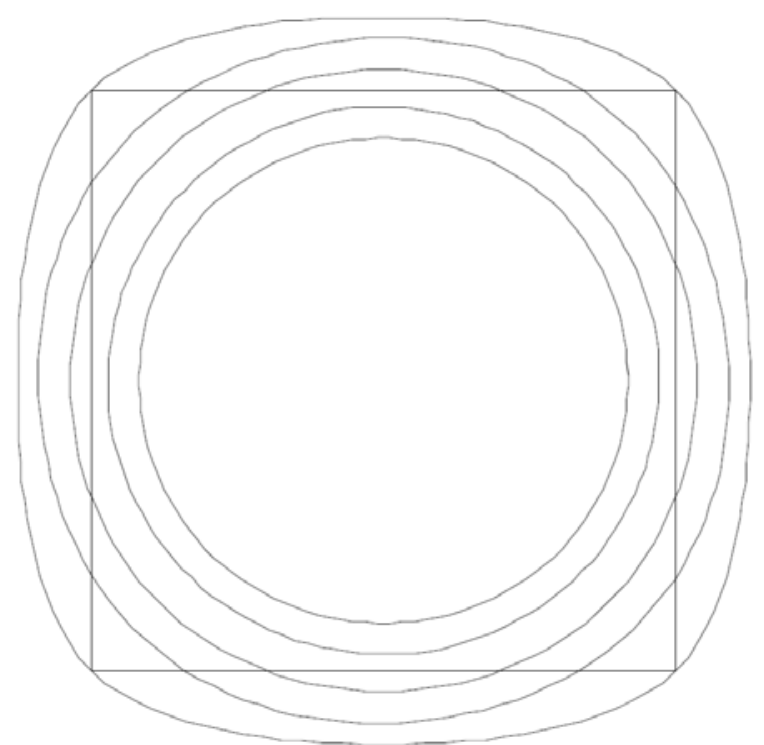

Figure 1. A new five-point binary subdivision scheme with a parameter (outside to inside: $t=0,0.4,0.5,0.6,1$ ). 


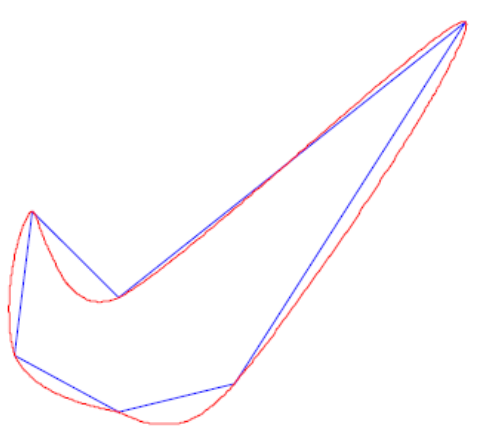

(a) $t=0$

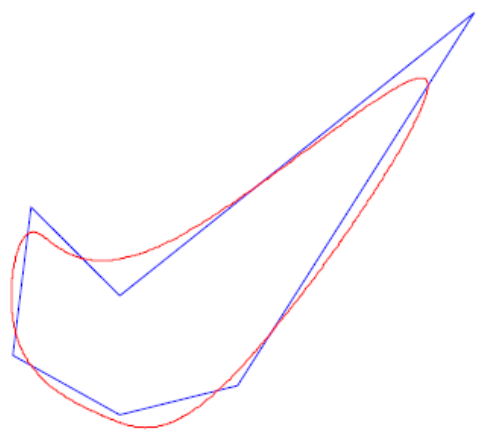

(c) $t=0.4$

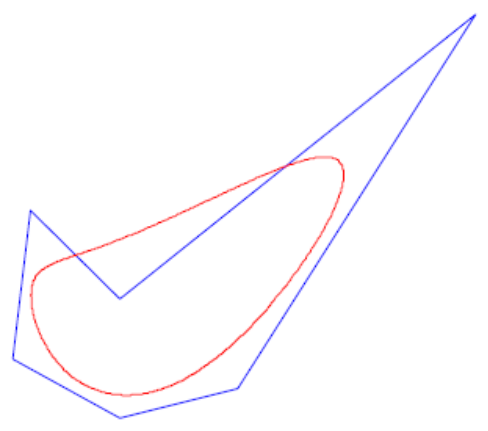

(e) $t=0.75$

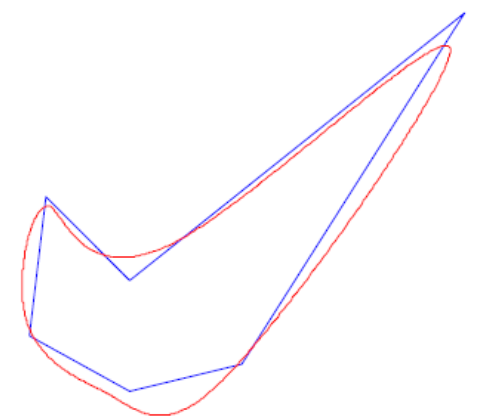

(b) $t=0.25$

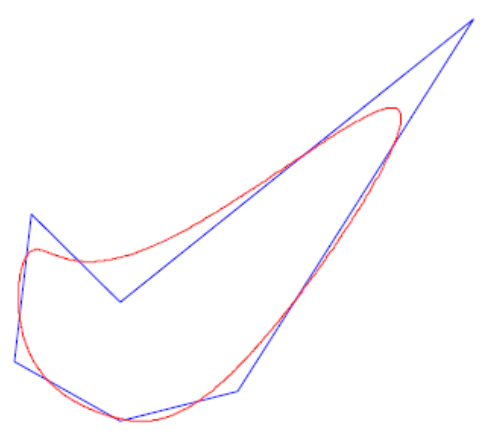

(d) $t=0.5$

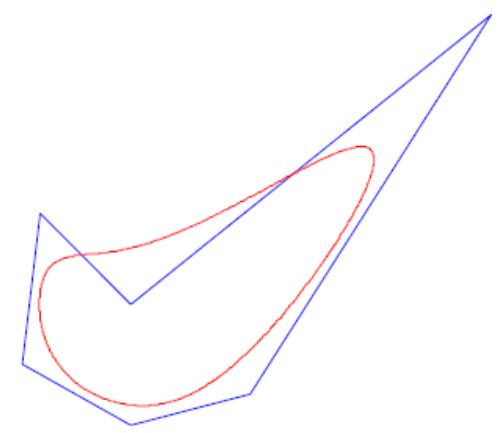

(f) $t=1$

Figure 2. A new five-point binary subdivision scheme with a parameter $(t=0,0.25,0.4,0.5,0.75,1)$. 


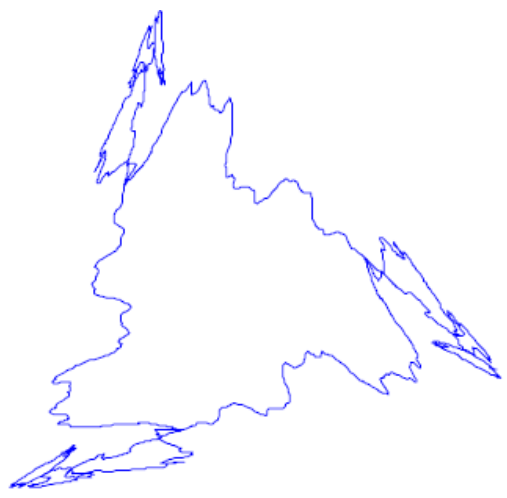

(a) $t=-1$

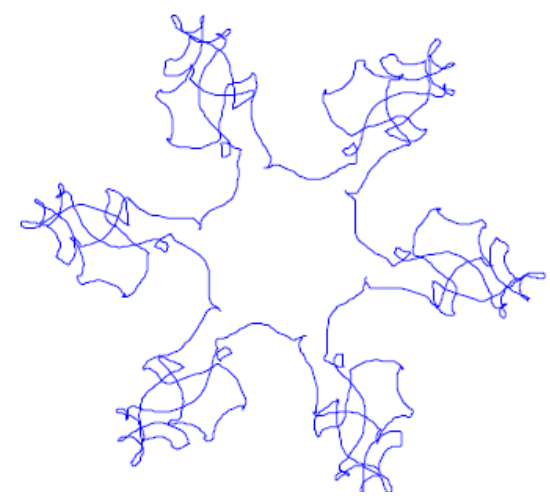

(b) $t=2.35$

Figure 3. Fractal curves.

\section{Numerical Example and Conclusions}

In this section, three examples are depicted in Figures 1, 2, and 3 to illustrate our scheme. In Figures 1 and 2, for the same initial control polygons, taking different values of parameter $t$, we can get different interpolation and approximation curves with different continuity.

In the process of mapping, we find when tension parameter $t$ take some special values, the limit curve will produce fractal behaviour. Figure 2 shows two examples of approximation of fractal limit curves produced by applying our subdivision scheme after six subdivision steps based on the same initial control polygon. The curves in Figure 2(a) and (b) are obtained with $t=-1$ and $t=2.35$, respectively.

In this paper, a new five-point binary subdivision scheme is presented and analyzed. We discussed the uniform convergence and continuity of this subdivision scheme by using generating polynomial method. The examples illustrate that our proposed scheme gives great flexibility to geometric designers when designing smooth curves according to their own requirements by choosing appropriate parameters. One of our further work will be aimed at the study of the conditions of the scheme's convexity preservation when lies in certain negative interval. Extending the curves subdivision scheme to surfaces is also our interested topic. 


\section{Acknowledgements}

This work is supported by the Special Funds for Young Scholars of Hefei Normal University under Grant No. 2017QN42. Special thanks also go to the referees.

\section{References}

[1] S. Dubuc, Interpolation through an iterative scheme, Journal of Mathematical Analysis and Applications 114(1) (1986), 185-204.

DOI: https://doi.org/10.1016/0022-247X(86)90077-6

[2] N. Dyn, D. Levin and J. A. Gregory, A 4-point interpolatory subdivision scheme for curve design, Computer Aided Geometric Design 4(4) (1987), 257-268.

DOI: https://doi.org/10.1016/0167-8396(87)90001-X

[3] A. Weissman, A 6-Point Interpolatory Subdivision Scheme for Curve Design, Master's Thesis, Tel-Aviv University, 1989.

[4] M. F. Hassan and N. A. Dodgson, Ternary and Three Point Univariate Subdivision Schemes, in: Albert Cohen, Jean-Louis Merrien and Larry L. Schumaker (Editors), Curve and Surface Fitting: Sant-Malo 2002, Nashboro Press, Brentwood (2003), 199-208.

[5] M. F. Hassan, I. P. Ivrissimitzis, N. A. Dodgson and M. A. Sabin, An interpolating 4-point $C^{2}$ ternary stationary subdivision scheme, Computer Aided Geometric Design 19(1) (2002), 1-18.

DOI: https://doi.org/10.1016/S0167-8396(01)00084-X

[6] G. M. Chaikin, An algorithm for high-speed curve generation, Computer Graphics and Image Processing 3(4) (1974), 346-349.

DOI: https://doi.org/10.1016/0146-664X(74)90028-8

[7] N. Dyn and D. Levin, Subdivision schemes in geometric modelling, Acta Numerica 11 (2002), 73-144.

DOI: https://doi.org/10.1017/S0962492902000028

[8] H. Zheng, Z. Ye and H. Zhao, A class of four-point subdivision schemes with two parameters and its properties, Journal of Computer-Aided Design and Computer Graphics 16(8) (2004), 1140-1145 (in Chinese).

[9] J. Tan, X. Zhuang and L. Zhang, A new four-point shape-preserving $C^{3}$ subdivision scheme, Computer Aided Geometric Design 31(1) (2014), 57-62.

DOI: https://doi.org/10.1016/j.cagd.2013.12.003 